\title{
Circulating cytokeratin 18 fragments and activation of dormant tumor cells in bone marrow of cancer patients (Review)
}

\author{
CHRISTOPH AUSCH, VERONIKA BUXHOFER-AUSCH，ULRIKE OLSZEWSKI and GERHARD HAMILTON
}

\author{
Ludwig Boltzmann Cluster of Translational Oncology, A-1090 Vienna, Austria
}

Received August 12, 2009; Accepted October 7, 2009

DOI: 10.3892/etm_00000002

\begin{abstract}
In cancer patients detection of systemic disease is of great importance to obtain prognostic information and to guide therapy. Bone marrow (BM) seems to be a common homing tissue for the early spread of tumor cells from various epithelial tumors; however, verification of the prognostic significance of BM-disseminated tumor cells (BM-DTCs), is restricted to breast cancer so far. These cells may be dormant for a long time, and signals triggering their activation leading to recurrence remain to be characterized. A recent study involving metastatic breast cancer patients reported that the shortest disease-free survival is correlated with cytokeratin (CK)-negative BM aspirates and that CK-positive BM-DTCs correspond to dormant tumor cells. Soluble CK fragments in serum including CK18 and 19 (measured as TPS and CYFRA 21-1, respectively) and caspase-cleaved CK18 are widely used to monitor tumor progression and response to therapy, actually indicating proliferation and/or necrotic/apoptotic cell death. In order to assess the source of the CK fragments, we used determinations of CK18 and caspase-cleaved CK18 fragments in serum samples before and after radical tumor surgery in colon cancer patients. Elevated serum concentrations of CK18 were found to persist in patients with a high incidence of BM-DTCs, and high perioperative levels of caspase-cleaved CK18 fragments were detected in patients with early relapses, respectively. These results indicate that in some patients at increased risk of recurrence disseminated cell populations exist that are responsible for the release of the bulk of CK fragments after removal of the apparently nonmetastatic tumor. In good agreement with the results in metastatic breast cancer patients, release of CK18 or 19 fragments by BM-DTCs seem to indicate disseminated tumor cells mainly in a dormant state, whereas caspase-cleaved CK18 may indicate skipping of this latent phase and early progression. Therefore, caspase cleavage of CKs in intact tumor cells seems to accompany
\end{abstract}

Correspondence to: Dr Gerhard Hamilton, Ludwig Boltzmann Cluster of Translational Oncology, c/o Balderichgasse 26/13, A-1170 Vienna, Austria

E-mail: gerhard.hamilton@toc.lbg.ac.at

Key words: cytokeratin, cytokeratin 18, cancer, disseminated tumor cell, bone marrow, epithelial-mesenchymal transition, prognosis or is involved in the differentiation leading from dormant to progressively active disseminated tumor cells. Release of respective CK fragments would result in an apparent clearing of CK-positive cells in BM, leaving malignant cells that have possibly undergone an epithelial-mesenchymal transition. Micrometastatic cancer cell lines derived from breast cancer patients were found to display loss of epithelial CK8, 18 and 19 as well as ectopic expression of vimentin as in mesenchymal cells. In conclusion, degradation of CKs may represent a marker indicating reactivation of dormant tumor cells in BM.

\section{Contents}

1. Introduction

2. Cytokeratins as markers of proliferating or dying tumor cells?

3. Effect of radical tumor surgery on circulating cytokeratin 18 fragments in colon cancer

4. Release of cytokeratins by the tumor and/or disseminated tumor cells in colon cancer?

\section{Conclusion}

\section{Introduction}

Appearance of disseminated tumor cells (DTCs) in cancer patients may precede the occurrence of detectable metastases and, provided that their detection is predictive, may be used to adapt the aggressiveness of therapeutic interventions (1). Evidence for such a role of bone marrow BM-DTCs or circulating tumor cells has been confirmed for breast cancer patients, where BM-positive patients have an approximately 2-fold increased risk of relapse within 10 years; however, the situation is less clear for other solid tumors such as colon cancer $(1,2)$. BM-DTCs may survive for prolonged periods in a dormant state, leading to late tumor recurrences upon reactivation. Cytokeratins (CKs) are in use for the detection of epithelial cancer cells in BM with the help of pan-CK antibodies directed to CK8, 18, 19. Furthermore, these proteins seem to be of functional importance in BM-DTCs. Alix-Panabiéres et al demonstrated that full-length CK19 is released by viable epithelial tumor cells and that CK19releasing cells might constitute a biologically active subset of breast cancer cells with highly metastatic properties (3). CKs, 
belonging to the intermediate filament (IF) protein family, are particularly useful markers in oncology diagnostics (4). At present, more than 20 different CKs have been identified. CK8, 18 and 19 are the most abundant in simple epithelial cells and carcinomas, including those of the breast, prostate, lung, colon and ovary (5). Usually low weight, acidic type I CKs such as CK18, complex with high weight, basic or neutral type II CKs, such as CK8. The bulk of cellular CKs is part of the IF system and insoluble at physiological salt concentrations until cleaved to yield soluble fragments. Upon release from proliferating or necrotic/apoptotic cells, CKs provide useful markers for the ongoing cell activity of epithelial malignancies, distinctly reflecting tumor progression/recurrence or response to therapy $(4,6,7)$. The three most applied CK markers for the assessment of cell activity used in the clinic are tissue polypeptide antigen (TPA), tissue polypeptide-specific antigen (TPS) and CYFRA 21-1. TPA is a broad spectrum test that measures CK8, 18 and 19, while TPS and CYFRA 21-1 assays are more specific and measure CK18 and 19, respectively (6). Assessment of soluble CK fragments in serum samples was further refined by the availability of monoclonal antibodies to epitopes of soluble CK18 and caspase-cleaved CK18 (CK18-Asp396-NE), recognizing fragments of 65 and $30 \mathrm{kDa}$, respectively (Peviva, Bromma, Sweden).

\section{Cytokeratins as markers of proliferating or dying tumor cells?}

Originally, TPA was described as an antigenic molecule that is generally present in human carcinomas and released by proliferating cells (8). In cultures of human HeLa cells, perinuclear formation of TPA was observed early in the S phase, and after cell division TPA was found to be externalized. Proliferating cells have a substantial pool of soluble $\mathrm{CK} 8$ and 18 , and $\mathrm{G}_{2}-\mathrm{M}$ arrest induces an increase in this pool (9). Although data regarding cell death are lacking in some of these reports, it seems reasonable to assume that viable cells were used in the experiments. The findings together with extensive clinical studies have led to the hypothesis that TPA is related to proliferative activity in general, especially in transformed epithelial cells. Similar to TPA, serum fragments of CK18 and 19 (measured as TPS and CYFRA 21-1, respectively) have traditionally been considered as markers of tumor proliferation, although evidence is scarce for a causative relationship between proliferation and levels of TPS and CYFRA 21-1 (10). Although the levels of CKs in serum of cancer patients have been widely used for monitoring the progression of cancer growth and the effectiveness of cancer treatment, the mechanisms involved in the release of CK fragments from cells have not been elucidated (11). Studies have shown that the release of CKs by tumors in patients is a complex process which is not simply correlated to the number of proliferating cells or to tumor mass and may be dependent also on the rate of cell damage (10).

During the development of CK18 as a tumor marker, the focus switched from the concept of this CK as a proliferation marker to its significance as a marker of tumor cell death $(10,12)$. Type I CKs (CK18 and 19) are cleaved by caspases during apoptosis, and the resulting fragments are relatively stable (13). Therefore, release of CK18 fragments was attrib- uted to tumor cell necrosis for soluble CK18 and apoptosis for caspase-cleaved CK18 (CK18-Asp396-NE). Furthermore, release of TPS and CYFRA 21-1 from tumor cells into the extracellular space has been demonstrated to occur during apoptosis (10). However, in a study involving human lung cancer cell lines, inhibition of caspase-3 resulted only in the partial inhibition of the release of CYFRA 21-1 (13). The demonstration of elevated levels of both caspase-cleaved and native CK18 in local tumor venous blood of endometrial cancer patients was taken as evidence that these proteins were derived from tumor tissue (12). Accordingly, test systems assessing total and caspase-cleaved CK18 are currently in use to determine response to chemotherapy in cancer patients and to distinguish necrotic and apoptotic tumor cell death. The concept involving the release of CKs from cells as an apoptotic or necrotic tumor cell death-associated mechanism no longer includes the early evidence of the production and secretion of these proteins as proliferation antigens by intact cells (6). Additionally and in contrast to this common view that CKs are only released in fragmented forms by epithelial cells as a result of necrosis/apoptosis, evidence for the release of full-length CK19 by viable epithelial tumor cells in an active export process was provided in a recent report (3). In this study, CK19-releasing cells were detectable in BM of 44-70\% of breast cancer patients and were correlated to the presence of overt metastases. In conclusion, regulation of the release of CK18/19 by intracellular (caspase-mediated) cleavage and transmembrane transport in intact/necrotic/apoptotic cells under conditions of cell proliferation and damage remains to be fully characterized.

\section{Effect of radical tumor surgery on circulating cytokeratin 18 fragments in colon cancer}

We quantified CK18 (M65) and caspase-cleaved CK18 (M30) in serum samples of colon cancer patients pre- and postoperatively (62 and 31 patients, respectively) using ELISA assays for the first time (14). Both low-grade tumors and disseminated metastatic colon cancers yielded higher levels of circulating CK18 and caspase-cleaved CK18 compared to nontumor control patients. Upon tumor surgery approximately $60 \%$ of the patients responded with a mean decrease of $30 \%$ in soluble CK18 while the remaining $40 \%$ of the patients had a mean increase of $40 \%$ in CK18 (15). The group with increased release of CK18 was characterized by a higher incidence of BM-DTCs in comparison to patients with normalizing CK18. In the case of circulating caspase-cleaved CK18, almost $80 \%$ of the patients exhibited a significant mean decrease of approximately $75 \%$ in response to tumor removal; however, the remaining $20 \%$ of the patients revealed lower basal levels of caspase-cleaved CK18 preoperatively and a mean increase of $40 \%$ in these CK fragment levels postoperatively (14). Tumors of a size of 2-6 cm were radically removed in those latter patients. The frequency of BM-DTCs, as detected with the aid of the pan-cytokeratin antibody A45-B/B3, was not significantly different in these two groups of patients with normalizing and increased caspase-cleaved CK18. However, caspase-cleaved CK18 correlated significantly with an increased number of recurrences within three years in the group with persistent serum fragment levels (hazard ratio 8.3). 
Only 4 of 11 patients exhibited a perioperative increase in circulating CK18 and caspase-cleaved CK18 at the same time. In conclusion, radical removal of the tumor, the supposed main source of the circulating $\mathrm{CK}$ fragments, failed to result in a decrease in CK18 levels to normal values in this subgroup of patients, characterized by BM-DTCs and increased risk of early recurrences.

In the same group of 62 colon cancer patients, the overall rate of BM-DTCs was $23 \%$ with a similarly positive fraction through all stages of the disease (16). After a median follow-up of 35.4 months, no differences were found in relapse and overall survival between patients with and without BM-DTCs preoperatively, while the BM-DTC status was found to be changed for a second $\mathrm{BM}$ aspiration after 12 months in a quarter of the patients.

\section{Release of cytokeratins by the tumor and/or disseminated tumor cells in colon cancer?}

Increased levels of circulating CK18 fragments in low grade and metastatic colon cancer patients may be associated with increased proliferation rather than increased cell death, since increased tumor progression is expected at these tumor stages. Removal of the tumor in colon cancer patients resulted in a reduction in circulating $\mathrm{CK}$ fragments in the majority of patients; however, in the remaining group persistent or increased perioperative levels of CK18 and caspase-cleaved CK18 point to disseminated tumor cells as a putative source of CK fragments (14). These same patients were characterized by either a high frequency of BM-DTCs or early relapses, respectively, in comparison to the patients with a perioperative reduction in circulating $\mathrm{CK}$ fragments.

According to a report by Bidard et al, BM-DTCs (CK8-, 18- or 19-positive epithelial cells) may undergo dormancy for several years before growing (or not) into clinically detectable metastases (17). In breast cancer patients with a metastatic relapse, BM-DTC-negative patients (approximately $40 \%$ of the patients) had a significantly shorter metastasis-free interval than BM-DTC-positive patients. Bidard et al hypothesized that a faster metastatic process, involving dissemination of DTCs to other host organs and/or epithelial-mesenchymal transition (EMT) from CK-positive to CK-negative DTCs, might skip the dormancy step. This hypothesis seems to corroborate our CK18 measurement results in colon cancer patients: postoperative release of CK19 by disseminated tumor cells is correlated with a higher incidence of BM-DTCs and elevated release of circulating caspase-cleaved CK18 with early relapses (14). These increases in the latter fragments may indicate generation of CK-negative BM-DTCs that initiate relapses in colon cancer patients, similar to breast cancer patients.

Several possible mechanisms could be responsible for the generation of circulating caspase-cleaved CK18 in patients with early relapses following radical tumor surgery. Circulating tumor cells released during surgery may undergo apoptotic tumor cell death due to failure of homing of the majority of cells to suitable environments, while a minor fraction eventually manages to settle at distant sites leading to tumor recurrence (18). Retsky et al suggested that surgery to remove the primary tumor often terminates dormancy resulting in accelerated relapses in over half of all metastatic cases (19). Another explanation may be intracellular degradation of CK18 by caspases and release into the circulation as part of the EMT without cell death or, alternatively, secretion of CK18 in an intact form, as shown for CK19, followed by its cleavage by caspases in the BM or serum, since the observed increased risk of tumor progression is in disaccord with the apoptotic death of the disseminated tumor cells (20). Caspases, apart from their role in apoptotic cell death, seem to have important functions in regulating differentiation in various cell types, including cells of the bone $(21,22)$. Although the disappearance of CKs was demonstrated in breast cancer micrometastatic cells isolated from BM by proteomics and immunohistochemistry, the mechanism of the downregulation of their expression has not yet been elucidated (23).

\section{Conclusion}

New evidence indicates that disseminated cancer cells in breast and colon tumors proceed from a dormant to a malignant state upon changes in their cellular phenotypes, including remodeling of the cytoskeleton and downregulation of the expression of CKs. It is hypothesized that this epithelial-mesenchymal-like transition is affected, at least in part, by apoptotic degradation of type I CK18 and 19 and release of the stable fragments by intact cells, or cell-free degradation of soluble CK18/19 secreted by the cells. If valid, this establishes caspase-cleaved CK18 as a marker of malignity of BM-DTCs in tumor patients following radical surgery. This could be tested by studying the fate of CKs in micrometastatic cells to determine whether they are removed as intact soluble fragments or released following proteolytic degradation and by investigating the association of early relapses and circulating caspase-cleaved CK18 in larger populations of patients following radical tumor surgery.

\section{References}

1. Pantel K, Brakenhoff RH and Brandt B: Detection, clinical relevance and specific biological properties of disseminating tumour cells. Nat Rev Cancer 8: 329-340, 2008.

2. Fehm T, Müller V, Alix-Panabières $\mathrm{C}$ and Pantel $\mathrm{K}$ : Micrometastatic spread in breast cancer: detection, molecular characterization and clinical relevance. Breast Cancer Res 10: 1, 2008.

3. Alix-Panabieres C, Vendrell JP, Slijper M, Pelle O, Barbotte E, Mercier G, Jacot W, Fabbro M and Pantel K: Full length cytokeratin-19 is released by human tumor cells: a potential role in metastatic progression of breast cancer. Breast Cancer Res 11: 39, 2009.

4. Barak CV, Goike H, Panaretakis KW and Einarsson R: Clinical utility of cytokeratins as tumor markers. Clin Biochem 37: 529-540, 2004.

5. Coulombe PA and Omary MB: 'Hard' and 'soft' principles defining the structure, function and regulation of keratin intermediate filaments. Curr Opin Cell Biol 14: 110-122, 2002.

6. Linder S: Cytokeratin markers come of age. Tumor Biol 28: 189-195, 2007.

7. Scott LC, Evans TR, Cassidy J, Harden S, Paul J, Ullah R, O'Brien V and Brown R: Cytokeratin 18 in plasma of patients with gastrointestinal adenocarcinoma as a biomarker of tumour response. Br J Cancer 101: 410-417, 2009.

8. Björklund B and Björklund V: Specificity and basis of the tissue polypeptide antigen. Cancer Detect Prev 6: 41-50, 1983.

9. Stigbrand T: The versatility of cytokeratins as tumor markers. Tumour Biol 22: 1-3, 2001.

10. Sheard MA, Vojtesek B, Simickova M and Valik D: Release of cytokeratin-18 and -19 fragments (TPS and CYFRA 21-1) into the extracellular space. J Cell Biochem 85: 670-677, 2002. 
11. Oehr P, Vacata V, Ruhlmann J and Rink H: Computer modeling of cytokeratin release in clinical oncology. Anticancer Res 17: 3111-3112, 1997.

12. Kramer G, Erdal H, Mertens HJ, Nap M, Mauermann J, Steiner G, Marberger M, Bivén K, Shoshan MC and Linder S: Differentiation between cell death modes using measurements of different soluble forms of extracellular cytokeratin 18. Cancer Res 64: 1751-1756, 2004.

13. Dohmoto K, Hojo S, Fujita J, Yang Y, Ueda Y, Bandoh S, Yamaji Y, Ohtsuki Y, Dobashi $\mathrm{N}$, Ishida $\mathrm{T}$ and Takahara $\mathrm{J}$ : The role of caspase 3 in producing cytokeratin 19 fragment CYFRA21-1 in human lung cancer cell lines. Int J Cancer 91: 468-473, 2001.

14. Ausch C, Buxhofer-Ausch V, Olszewski U, Hinterberger W, Ogris E, Schiessel R and Hamilton G: Caspase-cleaved cytokeratin 18 fragment (M30) as marker of postoperative residual tumor load in colon cancer patients. Eur J Surg Oncol, Feb. 28 , 2009 (Epub ahead of print).

15. Ausch C, Buxhofer-Ausch V, Olszewski U, Schiessel R, Ogris E, Hinterberger W and Hamilton G: Circulating cytokeratin 18 fragment M65 - a potential marker of malignancy in colorectal cancer patients. J Gastrointest Surg, Sept. 2, 2009 (Epub ahead of print).

16. Buxhofer-Ausch V, Ausch C, Kitzweger E, Mollik M, ReinerConcin A, Ogris E, Stampfl M, Hamilton G, Schiessel R and Hinterberger W: Spontaneous changes in tumour cell dissemination to bone marrow in colorectal cancer. Colorectal Dis, May 18, 2009 (Epub ahead of print).
17. Bidard FC, Vincent-Salomon A, Sigal-Zafrani B, et al: Time to metastatic relapse and breast cancer cell dissemination in bone marrow at metastatic relapse. Clin Exp Metastasis 25: 871-875, 2008.

18. Weitz J, Kienle P, Lacroix J, Willeke F, Benner A, Lehnert T, Herfarth C and von Knebel Doeberitz M: Dissemination of tumor cells in patients undergoing surgery for colorectal cancer. Clin Cancer Res 4: 343-348, 1998.

19. Retsky MW, Demicheli R, Hrushesky WJ, Baum M and Gukas ID: Dormancy and surgery-driven escape from dormancy help explain some clinical features of breast cancer. APMIS 116: 730-741, 2008.

20. Mani SA, Guo W, Liao MJ, Eaton EN, Ayyanan A, Zhou AY, Brooks M, Reinhard F, Zhang CC, Shipitsin M, Campbell LL, Polyak K, Brisken C, Yang J and Weinberg RA: The epithelialmesenchymal transition generates cells with properties of stem cells. Cell 133: 704-715, 2008.

21. Abraham MC and Shaham S: Death without caspases, caspases without death. Trends Cell Biol 14: 184-193, 2004.

22. Launay S, Hermine O, Fontenay M, Kroemer G, Solary E and Garrido C: Vital functions for lethal caspases. Oncogene 24: 5137-5148, 2005.

23. Willipinski-Stapelfeldt B, Riethdorf S, Assmann V, et al: Changes in cytoskeletal protein composition indicative of an epithelialmesenchymal transition in human micrometastatic and primary breast carcinoma cells. Clin Cancer Res 11: 8006-8014, 2005. 